\title{
A Study of Hair Damage by Magic Straight Perm
}

*Correspondence to:

Lim SN,

Tel: +82-62-432-6685

Fax: +82-62-268-5235

E-mail: isn6685@nate.com

Received August 3, 2012

Revised September 8, 2012

Accepted September 14, 2012

\author{
Sun Nye Lim* \\ Department of Beauty Design, Dongshin University, Naju 520-714, Korea
}

In this study, the changes in hair quality before and after Magic straight perm have been evaluated through a hair damage measurement method. For this, a healthy high school student's (age18 years) wavy hair was selected and permed on the left and right sides. Then, the changes caused by physical methods which were applied during the flat ironbased Magic straight perm were evaluated based on the hair damage measurement method before and after the Magic straight perm. According to the protein release test after the Magic straight perm, $1.26 \%$ in average and $0.14 \%$ was observed in Cool Magic straight perm sample. In a field emission scanning electron microscopy (FE-SEM) test, saw teethshaped partial desquamation of cuticle cells and impurities were observed in the warmtreated hair sample. In atomic force microscope (AFM), line-profile is a method to represent roughness data on hair. According to analysis on 3-dimensional (3D) images, the hair with Cool Magic straight perm was lower than the hair with Warm Magic perm in terms of the color change of 3D images. In addition, vertical changes were observed in the hair with Cool Magic perm. As a result, irregular surface roughness was observed. This study proposed a method to minimize hair damage by cooling down the heat with the cool hair straightener as soon as the Warm Magic was finished.

Key Words: AFM, Cool Magic straight, FE-SEM, Protein release, Line-profile, 3D image

\section{INTRODUCTION}

In modern times, aesthetic purpose has diversely changed. People of all ages and genders have become more interested in hair styles, and in turn hair damage has further increased. Keratin protein fibers in hair are influenced by routine hair care, chemical treatment and environmental factors in terms of chemical characteristics and physical strength. Even though hair surface may look smooth and glossy with the naked eye, it is actually covered by the scales which are formed by the cuticle cells when observed with field emission scanning electron microscopy (FE-SEM) (Lee \& Chang, 2010). In Korea, because those with curly hair account for $53 \%$ (25\%, inborn; $28 \%$, acquired) of total population, preference of straight hair has been high (Cho, 2007). In addition, it has been reported that most Korean people have curly hair (Lee \& Kang, 2008). In terms of Magic straightening tool, flat iron is generally used to straighten the curly hair (Kang et al., 2003).
A flat iron is a hair straightening tool which is commonly used to straighten curly hair. The tong-flat iron is made of a pair of metal or ceramic plates which are wrapped by plastic case. A metal plate can be heated up to an optimal temperature (Breuning \& Papini, 2008). Even though the Magic straight perm has satisfied a lot of people, a problem of hair damage caused by the inconsiderate use of perm agent or hair straightener has increased. The hair straightener which is as hot as over $180^{\circ} \mathrm{C}$ treats hair, and Magic straight perm cream which is used as reducing agent is high in terms of alkalinity. Therefore, even though it may look glossy and healthy hair, just one treatment can cause severe hair damage (severer than the damage caused by general perm chemicals) (Kim, 2004). In the 1980s, the perm which used a special solution (mixture of wheat flour with perm wave solution and straight perm agent) was popular. Entering the 1990s, it started to lose its popularity because it was not as good as the flat ironbased Magic straight perm in terms of hair straightening

@ This is an open-access article distributed under the terms of the Creative Commons Attribution Non-Commercial License (http://creativecommons.org/licenses/by-nc/3.0) which permits unrestricted noncommercial use, distribution, and reproduction in any medium, provided the original work is properly cited.

Copyrights (C 2012 by Korean Society of Microscopy 
and glossiness. In general, hair is about $10 \sim 15 \%$ in terms of water content. If it is heated, it loses its moisture. In addition, it swells around $120^{\circ} \mathrm{C}$. If $130 \sim 150^{\circ} \mathrm{C}$ of heat is imposed, hair starts to lose its color, smell and deform. Bubbles are produced on the cortex and medulla, and hair starts to lose elasticity (Lim \& Song, 2011).

In terms of structure, hair consists of three layers; cuticle, cortex and medulla. In case of curly hair, ortho-cortex and para-cortex exist in the concentricity just like wool or dispersed. Otherwise, either o-cortex or p-cortex is found (Ryu, 2002). In other words, as o-cortex becomes bulky, it accounts for the outer part of the winding shape of the curly hair. Then, relatively solid p-cortex decreases in volume. As a result, hair gets curly. In case of curly hair, in particular, hair shaft gets twisted. Because hair diameter in the twisted part decreases, it becomes vulnerable to breakage. Therefore, hair greatly varies depending on the intensity of twisting, hair thickness and hair damage (Syed et al., 1995). Cuticle layer protects cortex, makes the hair smooth and soft and reduces friction between hair (Chang, 2003).

Cortex is an intermediate layer of hair, in which cornified cells are closely adhered to each other. It contains melanin which decides the color of hair (Shin et al., 2007). The medulla situated in the middle of hair contains hive-shaped air. It is related with hair glossiness and retaining heat. In fact, hair medulla is well developed in animals in cold regions (Seo, 2008). In particular, once the Magic straight perm is treated, the treated hair is kept straight permanently. If new hair grows, the Magic straight perm is treated to the new growth areas. Because people tend to grow their hair, however, they need to get the 2 nd and 3rd treatments on their hair which was already treated with Magic straight, which in turn causes another hair damage. In addition, re-treatment has often caused severe hair damage. The studies on hair damage can be classified into physical and chemical methods. The evaluation on hair damage in morphological abnormalities has been conducted by many researchers using FE-SEM. Damaged hair has been observed and classified using FESEM. In some studies, damaged hair has been coated with chitosan, and hair surface has been observed with FE-SEM (Shin \& Yoo, 2004). In addition, there have been studies on evaluation of ampule-treatment effects in the process of perming damaged dyed hair using FE-SEM (Park \& Choi, 2003). Atomic force microscope (AFM) has been used in the studies on the conditioning of the micro-structure analysis method of hair. As a result, it is necessary to conduct a study on Magic straight perm methods. In terms of thermal analysis on decrease in hair weight by the heating temperature of hair, Guthrie et al. (1995) performed comparative analysis on normal, dyed and bleached hairs. In addition, Kim et al. (2008) conducted thermal analysis on dyed hair while Rho et al. (2010) thermally analyzed the straight-permed hair. In terms of studies on AFM, furthermore, Hyun et al. (2008) only conducted a study on the conditioning effect of hair cosmetics.

In this study, the Magic straight perm was given to high school students with healthy hair (aged 18 years) using flat iron. Then, it was attempted to figure out a way to prevent hair damage during the Magic straight perm by investigating hair changes after Cool Magic and Warm Magic. In addition, each sample's shape has been observed using FE-SEM. Furthermore, the 3-dimentional (3D) information in scale structure on the surface of the sample has been measured using the AFM with which surface characteristics can be measured up to atomic unit. Therefore, this study is aimed to provide basic data for improvement of hair damage which can occur during hair treatment by analyzing physical changes of hair during the Magic straight perm through AFM.

\section{MATERIALS AND METHODS}

\section{Test Materials}

To observe hair changes by Magic straight perm, high school students' (aged 18 years) chemical treatment-free healthy hair has been sampled. Because results can differ depending on the students' holding force and a researcher's pulling force, the sample hair was directly permed and cut. After cutting the damaged hair by about $3 \mathrm{~cm}$, the sample hair was divided with back center. After blocking $5 \mathrm{~cm}$ in length and $20 \mathrm{~cm}$ in width to the left in the back center and giving Warm Magic perm, the Cool Magic straight perm was performed. Then, after blocking $5 \mathrm{~cm}$ in length and $20 \mathrm{~cm}$ in width to the right, Warm Magic straight perm was given. In terms of a perm method, after blocking, the sample hair was divided into 5 $\mathrm{cm}$ in length and $0.5 \mathrm{~cm}$ in width and permed. The hair was cut in $20 \mathrm{~cm}$ in length, about $10 \mathrm{~cm}$ off from the hair on the occipital region. The sampled hair was divided by $2 \mathrm{~g}$ in weight and fixed with silicon. Then, it was washed in distilled water and air-dried.

The agents used for the Magic straight perm include a creamtype agent for Magic straight perm only manufactured by Ion Renew Relaxer (Yedang Cosmetic, Incheon, Korea) (1st agent) and spray-type oxidizing agent (2nd agent). In terms of perm procedure, first, hair was washed using neutral shampoo and rinsed in distilled water. Then, it was wiped with tower and air-dried in cool air. After applying the 1st agent and giving thermal treatment for 10 minutes, the hair was left in room temperature for 10 minutes. To check how soft the hair is, the $4 \mathrm{~cm}$-long hair was picked up with two fingers, and a round was formed. Then, the elasticity was tested with the naked eye and touching sense. After washing the hair in running water and rinsing it in distilled water, it was wiped with a tower and dried in cool air using a hair dryer, keeping about $20 \mathrm{~cm}$ distance. Depending on a perm method, constant force was 


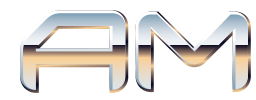

applied at $180^{\circ} \mathrm{C}$. Then, perm was done three times up to twothird of the hair length in the root and another three times up to the tip of the hair. The Magic straight-permed hair was coated with two drops of the perm agent and left in the air for 10 minutes. Then, it was washed in running water and dried in the air (ION RENEW RELAXER, Yedang Cosmetic, Korea).

\section{Equipment and Tools}

For the Magic straight perm, straight iron (XP-37; BS-Korea, Korea) and One-stop Beauty System (Hanaro-G, Korea) were used. To measure the temperature of Cool Magic straightener, an IR thermometer (ST-652, China) was used.

\section{Protein Release}

After performing the Magic straight perm, hair sample ( $0.25 \mathrm{~g})$ was immersed in $2.5 \% \mathrm{NaOH}$ solution $(50 \mathrm{~mL})$ for $10,20,30$, 40 and 60 minutes respectively. Then, the solution was filtered and measured at $340 \mathrm{~nm}$ using ultraviolet spectrophotometer (8452A Diode Array Spectrophotometer; Hewlett-Packard Asid Ltd., USA). After that, the amount of protein released from the calibration curve was evaluated.

\section{FE-SEM}

To observe the morphological changes of hair by the Magic straight perm, hair $5 \mathrm{~mm}$ in length has been sampled and arranged on the carbon-tape stub. Then, after platinumcoating the sample hair in $20 \mathrm{~nm}$ using IB-5 ion coater (Eiko, Japan), it was observed using FE-SEM (JSM-7500F + EDS, Oxford, Japan) at $15 \mathrm{kV}(\times 1,000, \times 5,000)$.

\section{AFM}

To observe the morphological changes of hair by the Magic straight perm using AFM, hair $5 \mathrm{~mm}$ in length has been sampled and arranged on the carbon-tape stub. Then, the sample hair was platinum-coated in $20 \mathrm{~nm}$ using IB-5 ion

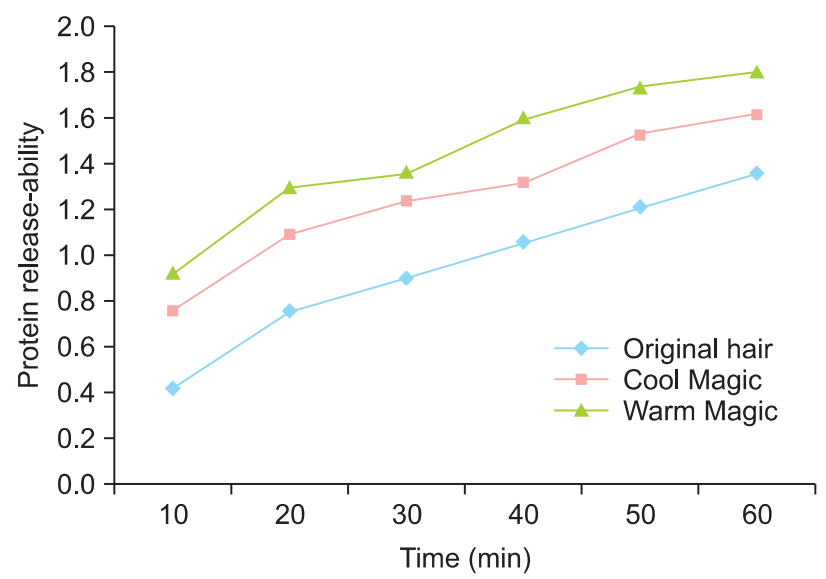

Fig. 1. Protein release before and after Magic Perm (original hair, Cool Magic, Warm Magic). coater (Eiko). In terms of AFM-based 3D hair structure, the surface structure of hair has been measured under the same conditions (length, $20 \mu \mathrm{m}$; width, $20 \mu \mathrm{m}$; height, $3 \mu \mathrm{m}$ ) using XE-100 (Park System, Korea).

\section{RESULTS AND DISCUSSION}

\section{Changes in Protein Release}

A test on protein release after the Magic straight is to evaluate hair damage. Specifically, hair damage was evaluated based on protein release before and after the perm and the amount of protein released after shampooing 10 times. When the heat was cooled down using the Cool Magic straightener, which is common in a beauty shop, protein release significantly declined. The average protein release of original hair was $0.95 \%$. It increased up to 1.65 (by $0.7 \%$ ) after shampooing 10 times. In other words, hair has been damaged by shampooing. In case of Cool Magic, the average protein release after the perm was $1.26 \%$. The measurement continued until 50 minutes passed since 10 times of shampooing (at $340 \mathrm{~nm}$ ). Since 50 minutes, however, it wasn't able to continue the measurement due to dramatic increase in protein release. It appears that protein release has significantly decreased because of contraction of cuticle when the heated hair was quickly cooled down with cool air. In addition, the average protein release right after the Warm Magic perm was $1.45 \%$. It was measured at $340 \mathrm{~nm}$ (until 10 minutes passed since 10 times of shampooing). Since 10 minutes, however, it wasn't able to continue the measurement due to dramatic increase in protein release (Fig. 1). After shampooing the Magic straight permed hair 10 times, dramatic protein release was observed (Fig. 2). In particular, when the Warm Magic perm was continuously given at $180^{\circ} \mathrm{C}$, hair damage was accelerated with significant increase in protein release.

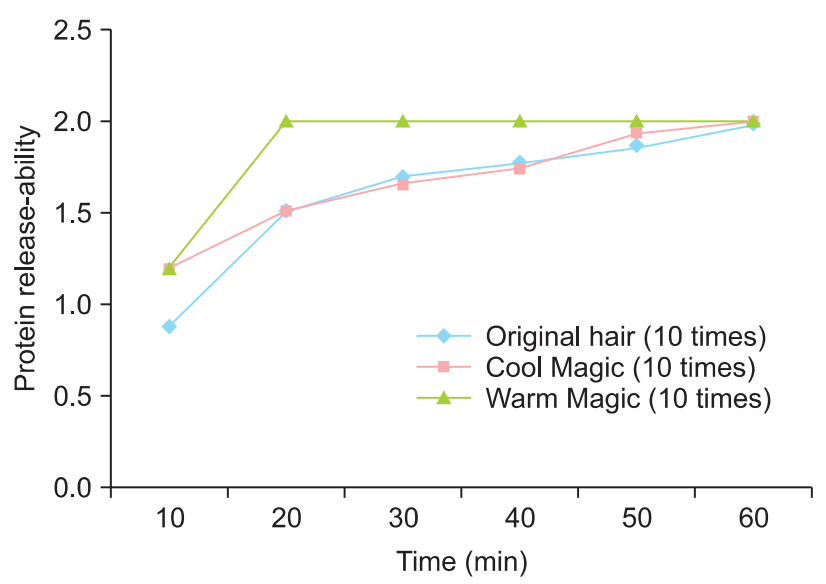

Fig. 2. Protein release after shampooing 10 times before and after Magic Perm (original hair, Cool Magic, Warm Magic). 


\section{Observation with FE-SEM}

To examine hair damage by the Magic straight perm, hair was sampled $5 \mathrm{~m}$ in length and observed with FE-SEM. In healthy hair, a cuticle layer arranges scale structure regularly, makes the hair glossy and protects the inside of the hair. In virgin hair, the surface was mostly gentle and smooth. In addition, cuticle was clearly found between scales. No loosening was found in damaged hair (Fig. 3). In the Cool Magic-permed sample, loosening was partially observed at the tip of the scale structure. No desquamation occurred (Fig. 4). In addition, cuticle cells were more severely separated in the Warm Magicpermed hair $\left(180^{\circ} \mathrm{C}\right)$ than the Cool Magic permed hair.
In addition, saw teeth-shaped desquamation was partially observed along with impurities (Fig. 5). Then, the scale in the saw teeth-shaped damaged part would fall off, and split ends could occur.

\section{Observation with AFM}

After fixing samples on the round metal disk with carbon tape, they were measured using AFM. Under the AFM method, the height of surface is measured using a probe (when it touches the surface). Using this method, the level of hair damage can be measured. After gluing the probe on the surface of the sample, the conditions of the hair surface were
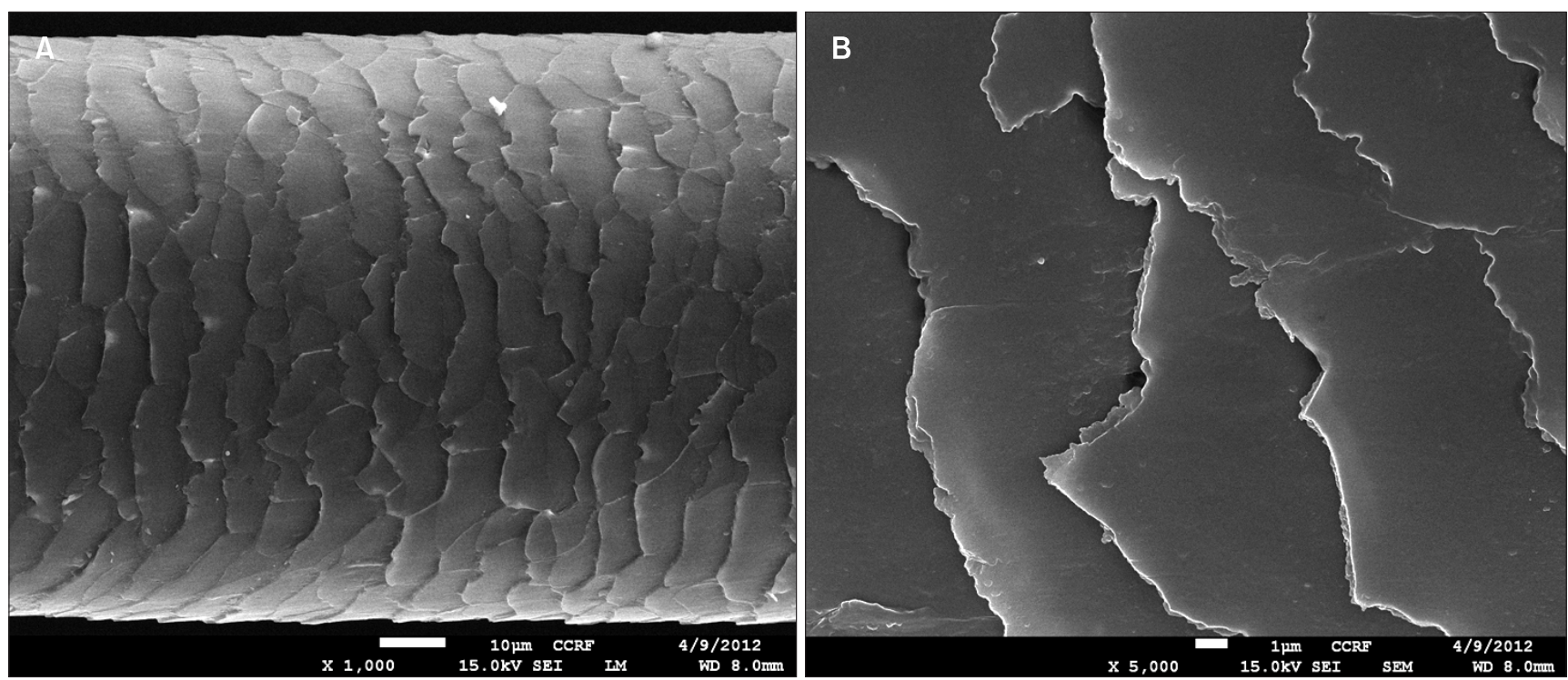

Fig. 3. Scanning electron microscope before treatment (original hair). (A) $\times 1,000,(B) \times 5,000$.
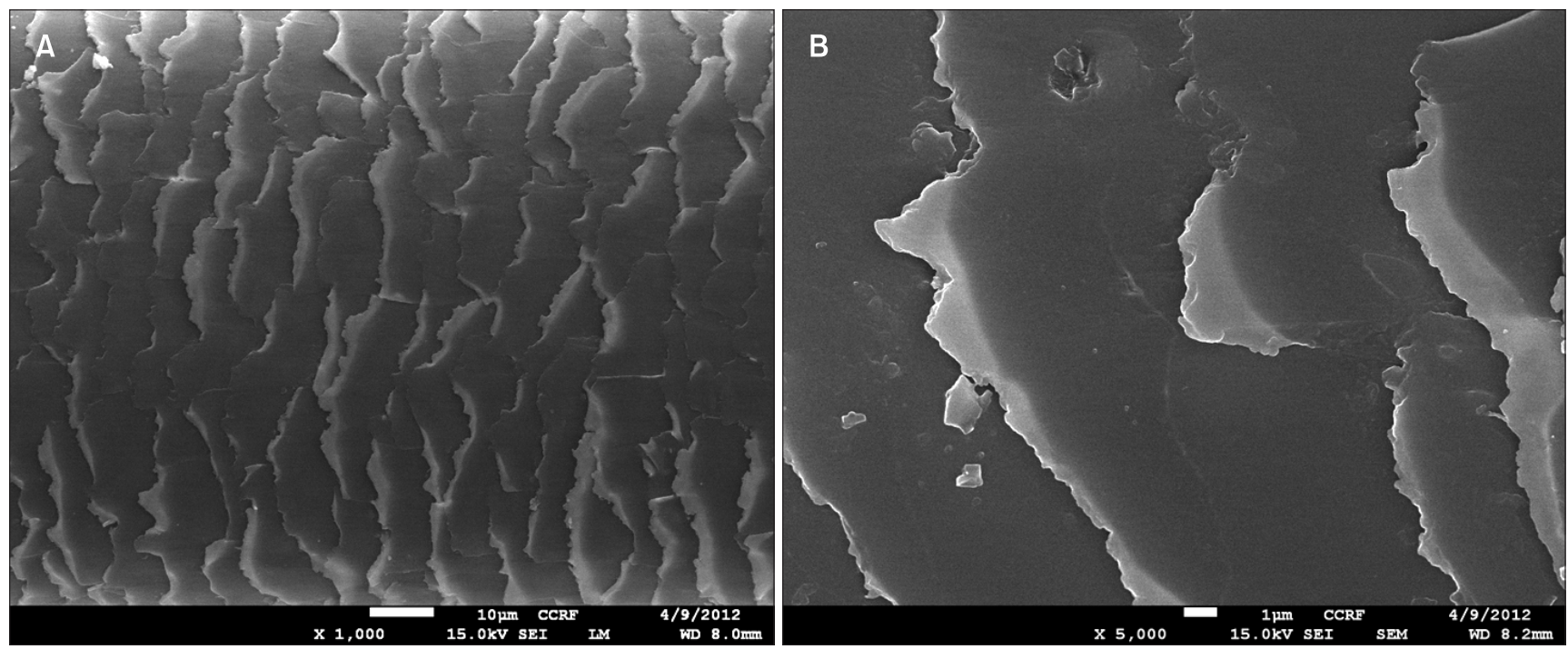

Fig. 4. Scanning electron microscope after Warm Magic Perm. (A) $\times 1,000,(B) \times 5,000$. 


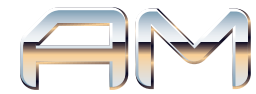

observed using the repulsive force which is vertically created on the surface. In the scale structure of hair, the protruded part is brightened while the dark area is concave. Based on the lightness of hair, the bright part represents a high area while the dark part means a low area. The line profile is the data which shows roughness after measuring height based on two inverted triangles depending on hair conditions. In 3D images, the line profile represents the roughness data of hair. It has been measured toward the cuticle of the sample. The data in a histogram ranged from -0.8 to $1 \mu \mathrm{m}$ with the height ranging from -4 to $0.4 \mu \mathrm{m}$. The line profile shows roughness data on hair. As shown in the 3D image analysis, the surface of cuticle of healthy hair was vivid in the results of the line profile as well. The roughness value between 8 and $12 \mu \mathrm{m}$ was $330 \mathrm{~nm}$. In addition, parallel angle has been confirmed based on the intrinsic hair axis (Fig. 6).

In the hair damaged by the Magic straight perm, the surface of cuticle was irregular in line profile data. In terms of roughness data, frequent height fluctuations based on the horizontal axis means several hair damage. The visual surface roughness
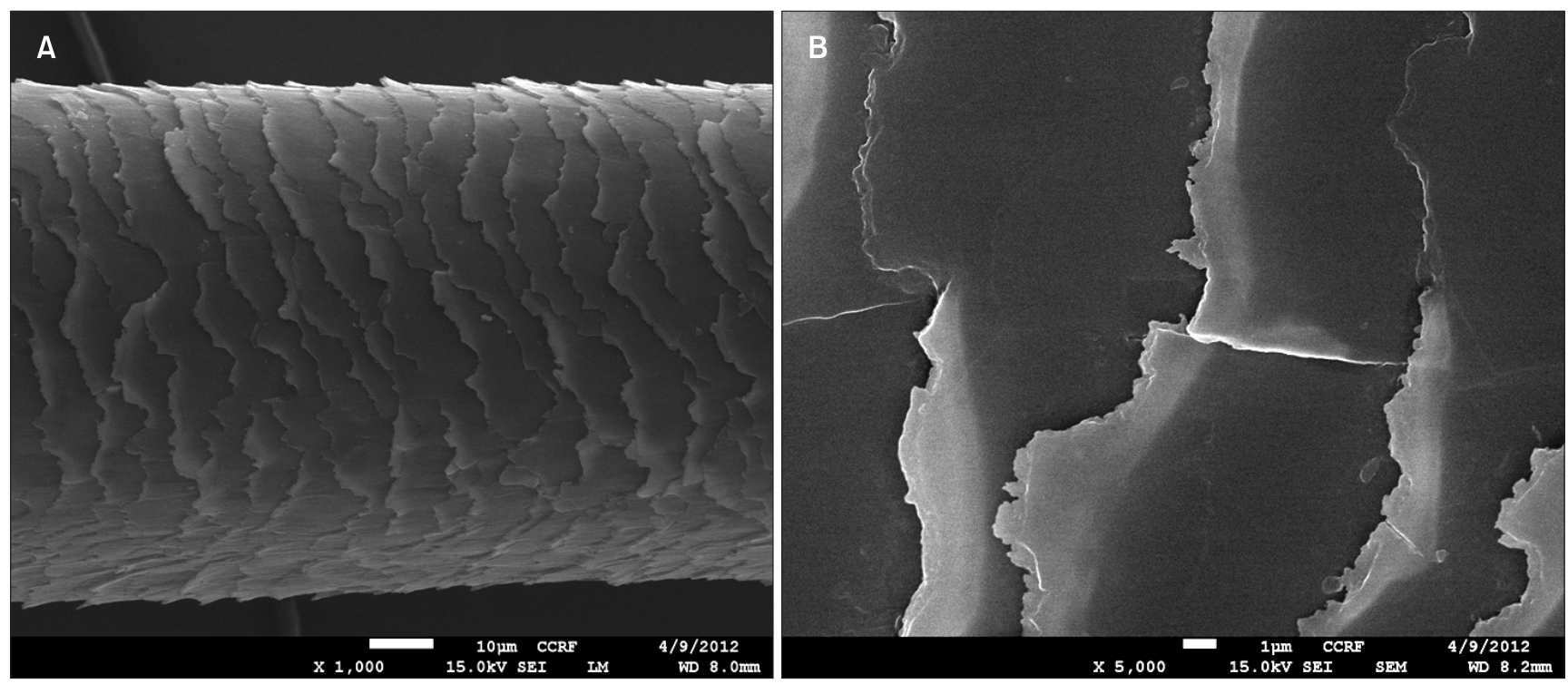

Fig. 5. Scanning electron microscope after Cool Magic Perm. (A) $\times 1,000$, (B) $\times 5,000$.
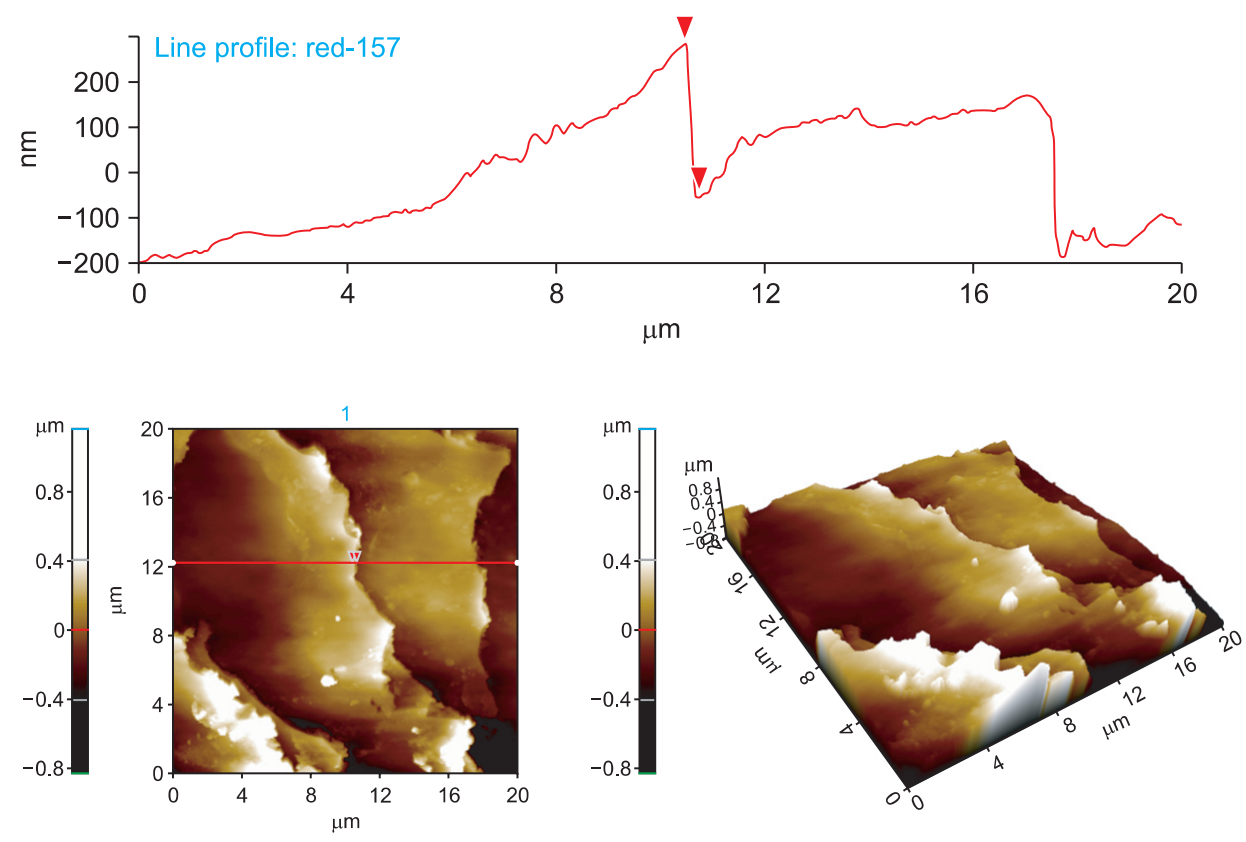

Fig. 6. Line profile and 3-dimensional images before original hair. 
of the damaged hair reveals in number. In case of the Magic permed hair at $180^{\circ} \mathrm{C}$, the roughness value at $8 \mu \mathrm{m}$ was 773 $\mathrm{nm}$. Loosening and partial breakage of cuticle were clearly observed because of desquamation of cuticles. Unlike healthy hair, in particular, frequent color changes in 3D image and irregular height fluctuations were observed in the common Warm Magic straight permed hair (Fig. 7).

After the Magic straight perm at $180^{\circ} \mathrm{C}$, the hair was rapidly cooled down using the Cool Magic straightener. In terms of roughness, fluctuations were not severer than the
Warm Magic permed hair. In the Cool Magic permed hair, roughness value between 12 and $16 \mu \mathrm{m}$ was $453 \mathrm{~nm}$. Even though cuticles were pretty loose, the surface was clean and smooth without breakage (Fig. 8). Unlike healthy hair, in particular, frequent color changes in 3D image and irregular height fluctuations were observed in the common Warm Magic straight permed hair.

In healthy hair, the border between cuticles was clear. After the chemical agent-used Magic straight perm, however, loose scales were observed on the surface of cuticles. In addition,
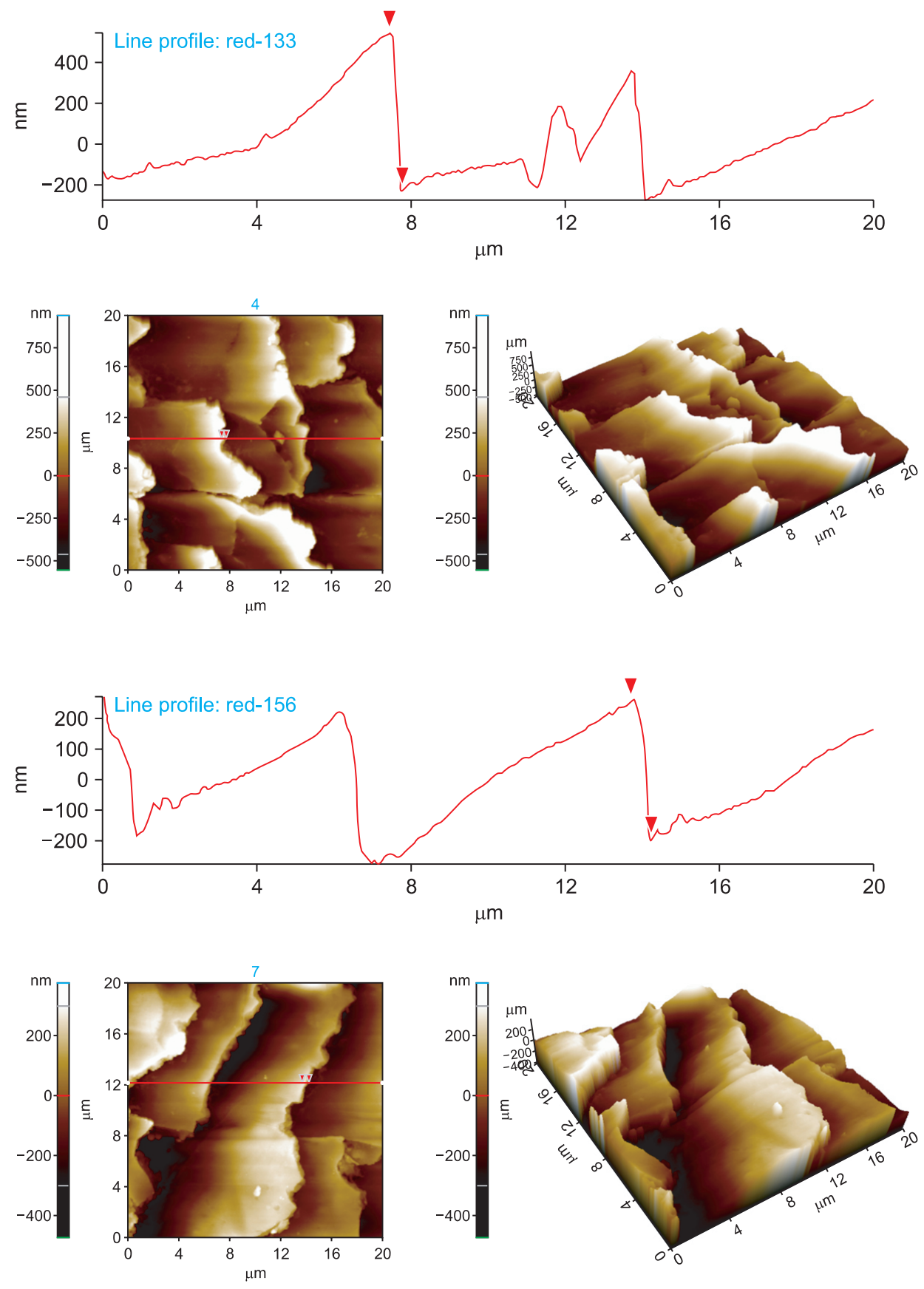

Fig. 7. Line profile and 3-dimensional image after Warm Magic.
Fig. 8. Line profile and 3-dimensional image after Cool Magic. 
scale split was detected due to high temperature.

\section{CONCLUSIONS}

Just as commonly done in a beauty shop, the Magic straight perm was done at $180^{\circ} \mathrm{C}$ in this study. After straightening the hair on the left at $180^{\circ} \mathrm{C}$, it was immediately cooled down using the Cool Magic straightener which was kept at $-10^{\circ} \mathrm{C}$ in a freezer. Then, hair damage was measured based on protein release, and the surface structure was observed with FE-SEM and AFM. In addition, the roughness data on the hair were observed using AFM. Then, the following results have been obtained:

1. According to the Cool Magic straight perm, protein release decreased. The decrease in protein release means that hair damage didn't occur any more because the surface of hair contracted during the Cool Magic perm right after the Warm Magic at $180^{\circ} \mathrm{C}$.

2. When the samples were observed with a microscope, the surface was smoother and cleaner on the Cool Magic permed hair than on the Warm Magic straight permed hair. 3. According to analysis on changes on hair surface using AFM, the Cool Magic permed hair was severer than the Warm Magic permed hair in terms of color changes and height fluctuations.

Based on the results above, it appears that hair damage could be minimized if hair is cooled down using the Cool Magic straightener right after the Warm Magic perm. It is also necessary to protect hair from chemicals and develop more efficient hair styling tools and equipment which can minimize hair damage through continued studies.

\section{REFERENCES}

Breuning E E and Papini R P G (2008) Hair straightener: a significant burn risk. Burns 34, 703-706.

Chang B S (2003) Fine structure of damaged hair shaft by daily treatment of heat for a beautiful face. Korean J. Electron Microscopy 33, 215-222.

Cho B S (2007) Personality and attitude of hair style om hair thickness from, Seo Kyeong University. Graduate School of Beauty Arts, pp. 1-10.

Guthrie J T, Kazlauciunas A, Rongong L, and Rush S (1995) The characterization of treated and dyed hair. Dyes and Pigments 29, 23-44.

Hyun J W, Lee K G, Yeo J H, and Tae B C (2008) A Study on the Conditioning Effect of the Hair Care Products Conditioning Silk Peptide. Korean J. Biotecnol. Bioeng. 23, 439-444.

Kang Y S, Soh Y J, Shim S N, Yoon S Y, Jang G G, and Jung S H (2003) Hair best permanent wave. Hunminsa, pp. 147.

Kim M S, Lee G Y, Choe E Y, Kim D H, and Chang B S (2008) Study on the thermal analysis of dyed hair depending on the brightness level. Korean J. Microscopy 38, 159-165.

Kim T G (2004) The study of morphological damage of hair in according with thermometer in the process of heat permanent. Master Thesis, The Graduate School of Health Science, Catholic University of Daegu, pp. 1-20.

Lee G Y and Chang B S (2010) General characteristics on the fine structure of scalp hair-I ultrastructure of hair shaft. Korean J.
Cosmetological Science 6, 265-271.

Lee H N and Kang S M (2008) The study on effects of curly hair by the permanent wave and day. Korean J. Fashion \& Beauty Society 6, 7579.

Lim S N and Song M R (2011) Scalp Trichology (Kwangmoonkag, Seoul), pp. 64-70.

Park J H and Choi J S (2003) The study of heat treatment effect before using ample on the permanent waving in heavy damaged coloring hair. Korean J. Society of Cosmetology 9, 88-95.

Seo Y Y (2008) Basic Hair Science (Yaelim, Seoul), pp. 21-27.

Shin H S and Yoo E K (2004) The observation of coating formation of damaged hair according to the chitosan treatment. Korean J. Society of Cosmetology 10, 1-6.

Shin J Y, Gwon D S, Kim H, Kim G R, Kim G S, Kim S H, Kim S H, Kim Y G, Na H S, No H S, Prak S K, Song H R, Yoon S Y, Jung J Y, Jin Y R, Cheon J C, Choi Y S, Ham M Y, and Hwang H J (2007) Beauty Care Cranc Introductiom (SooMoonSa, Seoul), pp. 115-122.

Syed A, Kuhajda A, Ayoub H, Ahmad K, and Frank E M (1995) AfricanAmerican hair: its physical properties and differences relative to Caucasian hair. Cosmet Toil 110, 39-48.

Rho J A, Lee G Y, and Chang B S (2010) Thermal analysis of the straight permed hair. Korean J. Microscopy 40, 163-168.

Ryu E J (2002) Trichology (Kwangmoonkag, Seoul), pp. 106-108. 